\title{
Mycophenolate mofetil for myasthenia gravis: a clear and present controversy
}

\author{
Chad Heatwole \\ Emma Ciafaloni \\ Department of Neurology, The \\ University of Rochester, Rochester, \\ New York, USA
}

\begin{abstract}
Mycophenolate mofetil (MMF) has been used to treat myasthenia gravis (MG) for over 10 years. MMF's use in the MG population stems from its theoretical mechanism of action and the medical literature that supports its benefit in MG patients. Recently, two large, double-blinded, placebo-controlled, randomized clinical trials were initiated to study the effectiveness of MMF for MG. One of these studies found no benefit in taking MMF with $20 \mathrm{mg}$ of prednisone as compared to taking prednisone alone, while the other study demonstrated no advantage in taking MMF against placebo during a 36-week prednisone taper. This article critically reviews the medical literature on MMF's use in MG and suggests further research avenues on this topic.
\end{abstract}

Keywords: myasthenia gravis, mycophenolate mofetil, CellCept

\section{Introduction}

Myasthenia gravis (MG) is an autoimmune disorder that affects the post-synaptic neuromuscular junction on skeletal muscle via autoantibody binding to the nicotinic acetylcholine receptors (AChR Ab). Antibody binding directly disrupts transmission between nerve and muscle fiber causing patients to experience fluctuating, fatigable weakness. Frequently, symptoms such as dysarthria, dysphagia, ptosis, and diplopia may occur. Occasionally, patients with MG may experience what is known as a myasthenia crisis: a lifethreatening respiratory compromise secondary to bulbar and diaphragmatic weakness.

The diagnosis of MG is based on the history and clinical findings; however, the presence of serum $\mathrm{AChR} A \mathrm{~b}$, a greater than $10 \%$ decrement during repetitive nerve stimulation, and an increased amount of jitter with single fiber electromyography (SFEMG) helps to confirm the diagnosis (Ciafaloni 2005).

The ideal treatment for MG should be affordable, applicable to all myasthenia patients, easily administered, $100 \%$ effective, and without adverse effects. Unfortunately, a therapy that meets all of these characteristics has yet to be discovered. While corticosteroids are the most commonly used therapy for MG, other immunosuppressing agents are also used: 1) In cases where MG is refractory to corticosteroid treatment; 2) As steroid-sparing agents; and, 3) In cases where severe corticosteroid side effects occur.

Currently available immunomodulating medications have varying mechanisms of action and side effect profiles. Immunomodulating medications frequently used for MG include: azathioprine, cyclosporine, cyclophosphamide, tacrolimus, intravenous immunoglobulin (IVIG), plasma exchange (PLEX) and mycophenolate mofetil. Thymectomy has also been identified as an immunomodulating therapy for MG, yet its true efficacy has yet to be definitively proven. The debate on the optimal agent for long-term management of myasthenia is far from settled.

\section{Mycophenolate mofetil}

Mycophenolate mofetil (MMF) (CellCept ${ }^{\mathbb{R}}$, Roche) is a synthesized pro-drug of mycophenolic acid that inhibits the immune system by preferentially depleting guanosine and 
deoxygunaosine on both $\mathrm{T}$ and B-lymphocyte lines (Allison and Eugui 2005). MMF is thought to selectively inhibit inosine monophosphate dehydrogenase type II, an enzyme that facilitates the production of an intermediate metabolite of guanosine (Schneider-Gold et al 2006). Ultimately, MMF is able to reduce the proliferation of $\mathrm{T}$ and B-lymphocytes and affect antibody formation and cell-mediated responses. MMF also acts on the immune system by: 1) Reducing lymphocytic recruitment to inflammation; 2) Limiting tissue-damaging nitric oxide; 3) Inhibiting the expression of adhesion molecules; 4) Reducing the secretion of tumor necrosis factor alpha; 5) Increasing the expression of interleukin-10; and, 6) Elevating the rate of lymphocytic apoptosis (Allison 2005; Schneider-Gold et al 2006). Given the unique immunosuppressive properties of MMF, it has been tried as a therapy for many autoimmune conditions including: lupus erythematosus, rheumatoid arthritis, systemic vasculitis, cerebral vasculitis, multiple sclerosis, MG, pemphigus vulgaris, psoriasis, inflammatory eye disease, Crohn's disease, Wegener's granulomatosis, dysimmune polyneuropathies, cluster headache, inflammatory myopathies, and organ transplantation (Epinette et al 1987; Enk and Knop 1997; Larkin and Lightman 1999; Neurath et al 1999; Nowack et al 1999; Meriggioli et al 2003; Rozen 2004; Cahoon and Kockler 2006; Schneider-Gold et al 2006).

Unlike most other classes of immunosuppressant therapy, MMF is not known to frequently produce major organ toxicity (Ciafaloni et al 2001). This feature of MMF makes it a potentially appealing therapy for MG. While cyclosporine and azathioprine may cause nephrotoxicity and hepatotoxicity respectively, these potentially severe side effects are rare to non-existent with MMF use (Lim et al 2007). MMF is also thought to have a lower rate of induced late malignancies than azathioprine and cyclophosphamide. In addition, compared with corticosteroids, MMF has fewer adverse effects on bone health, weight, cataract formation, and hypertension (Chaudhry et al 2001; Meriggioli et al 2003; Allison 2005).

The use of MMF does carry some risk. There is currently a FDA black box warning that indicates that the use of MMF may increase one's susceptibility to infection, the development of lymphoma, and may increase the risk of pregnancy loss and congenital malformation in childbearing woman. The most common side effect of MMF is gastrointestinal intolerance (usually diarrhea), however, nausea, vomiting, headaches, bone marrow suppression, sepsis, hypertension, tremor, neoplasia, depression, teratogenicity and an increased risk of infection may occur (Chaudhry et al 2001; Amato and Griggs 2003; Draper 2008). In one study of MMF combined with cyclosporine and corticosteroids for the prevention of acute renal transplant rejection, it was found that the MMF group (taking MMF at 1 or $1.5 \mathrm{~g}$ twice a day) had higher incidences (compared to a placebo group) of gastrointestinal symptoms, leucopenia, anemia, pancytopenia, agranulocytosis, cytomegalovirus tissue invasive disease, herpes zoster, herpes simplex and squamous-cell carcinoma (European Mycophenolate Mofetil Cooperative Study Group 1995).

Some severe adverse effects have also been reported in $\mathrm{MG}$ patients taking MMF. These effects have included: chronic heart failure, primary CNS lymphoma, a West Nile virus infection, a severe depressive episode, and a papulosquamous psoriatic-like skin eruption (Meriggioli et al 2003; Levin et al 2005; Vernino et al 2005; Draper 2008). Although the exact relationship between MMF and these adverse conditions has not been proven, close patient monitoring is certainly warranted for MG patients taking MMF.

The optimal dose of MMF for MG is also unknown. Typical dosing for neuromuscular disease ranges from $1500 \mathrm{mg}$ a day to $3000 \mathrm{mg}$ a day divided into twice a day (bid) or 3 times a day dosing. MMF has a high absorption rate from the gut and is $97 \%$ protein bound (Schneider-Gold et al 2006) Although plasma levels do not typically need to be checked, it is recommended to monitor complete blood cell counts routinely given the potential for leucopenia, anemia, pancytopenia, and agranulocytosis. While there is no direct contraindication in using MMF in conjunction with azathioprine, this practice is generally discouraged given the similar mechanism of action of these two medications.

\section{Review of MMF use for MG}

Up until 2008, the medical literature has largely been in favor of MMF for MG. Numerous case reports, an open-label pilot study, retrospective and studies, and one small placebo-controlled trial have all suggested clinical benefit. More recently, two double-blind, placebo-controlled, randomized clinical trials have produced less positive results. We will review the medical literature here.

To our knowledge, the first reported use of MMF for refractory MG was described in 1998 (Hauser et al 1998). In this report, a 26-year-old woman with MG manifesting with bulbar symptoms, weakness, and frequent hospitalizations was started on MMF at $500 \mathrm{mg} 3$ times a day. This patient noted benefits 5 days after receiving therapy. Her therapies prior to MMF included a transternal thymectomy, azathioprine for 6 months (discontinued secondary to nausea), multiple 
plasma exchanges, IVIG (stopped due to nausea and syncope), 10 sessions of 100 cGy total body irradiation, 6 years of cyclosporine, pyridostigmine, and oral steroids. At the time of MMF use, the patient was on cyclosporine ( $6 \mathrm{mg} / \mathrm{kg} /$ day), prednisone ( $1 \mathrm{mg} / \mathrm{kg} /$ day alternating with $2 \mathrm{mg} / \mathrm{kg} /$ day) and pyridostigmine ( $90 \mathrm{mg} /$ day). Cyclosporine was discontinued at the same time MMF was started given an increase in the patient's creatinine level. Benefits attributed to MMF at 15 months were a resolution in the patient's dysarthria, extremity weakness, shortness of breath, and a decrease in her hospitalization rate. In addition, while taking MMF, the patient was also able to reduce her pyridostigmine and prednisone dosages without adverse effects (Hauser et al 1998).

In 2000, a case report described the benefits of MMF in a woman with $\mathrm{MG}$ in her twenties (Meriggioli and Rowin 2000). Like the aforementioned patient, this woman had previously undergone a thymectomy and had both bulbar and extremity weakness. At the time MMF was added, she was on a regimen consisting of cyclosporine $(3.4 \mathrm{mg} / \mathrm{kg} /$ day), prednisone (40 mg/day of alternate-day treatment), and pyridostigmine $(360 \mathrm{mg} /$ day $)$. This patient had previously tried azathioprine, but had to discontinue it due to liver toxicity. After taking MMF for 3 weeks, this patient reported less fatigable weakness and after 11 months she was found to have improvement in her manual muscle testing and quantified MG score. Her requirement for prednisone, cyclosporine, and pyridostigmine also decreased (Meriggioli and Rowin 2000).

Given the promising findings of these case reports, in 2001, Ciafaloni et al (2001) published an open-label pilot study evaluating the use of MMF for MG. This study evaluated 12 patients between the ages of 18 and 80 with acquired MG. Enrolled patients had either refractory MG (to a regimen of prednisone and azathioprine or prednisone and cyclosporine) or they required additional medications after taking corticosteroids for eight months. MMF was given at a rate of $1 \mathrm{~g}$ every 12 hours for 6 months. The primary measures of efficacy were a reduction in the quantified $\mathrm{MG}(\mathrm{QMG})$ score and manual muscle test (MMT), or a reduction in corticosteroid dose for 3 months without worsening of QMG and MMT scores. Eleven of the 12 enrolled patients had a thymectomy 7 to 42 months prior to the study.

The results of this study revealed that 6 patients taking MMF had improved QMG and MMT scores while two patients taking MMF had significant reductions in their required corticosteroid dose. Of the 12 patients, one demonstrated a worsening of both QMG and MMT scores.
After 6 months, Wilcoxon signed-rank sum analysis demonstrated improvements in MMT, QMG, and activities of daily living compared with baseline $(\mathrm{p}=0.023,0.001$, 0.004 ) with treatment effects seen as early as two weeks. Single fiber EMG was also performed in this study population, but did not change with therapy. Some patients did experience side effects from MMF. Two patients developed a transient hand tremor and 2 patients were found to have a decreased hemoglobin level (although one was iron deficient). With the exception of these four patients, MMF was found to be well tolerated and safe (Ciafaloni et al 2001).

In 2001, a retrospective analysis of the use of MMF ( $1 \mathrm{~g}$ twice a day) for neuromuscular diseases was reported by Chaudhry et al (2001). Of the patients studied, 32 had MG (15 of which had previously undergone a thymectomy). Efficacy was defined as any improvement in functional status (including activities of daily living, ptosis, diplopia, facial weakness, bulbar weakness, arm abduction time, muscle strength, or FVC), or a reduction in the required dose of steroids by over $10 \mathrm{mg}$ a day. Of the $32 \mathrm{MG}$ patients, $22 \mathrm{had}$ improvements in either functional status or prednisone requirement with a time to improvement ranging from 2 to 12 months. Ten of the MG patients experienced no benefit after 8 months of use. In a subanalysis, patients that showed a favorable response to therapy tended to have been diagnosed with $\mathrm{MG}$ for a much shorter time (a mean of 7.5 months versus 14 years). Only 3 patients had been taking MMF as their only therapy in this study; the rest were taking combinations of corticosteroids, azathioprine, cyclosporine-A, methotrexate, plasma exchange and iv immunoglobulin. Some side effects were attributed to MMF. Three patients taking MMF developed gastrointestinal side effects and one other developed a "depressed mood" while on therapy (Chaudhry et al 2001).

In 2001, MMF was also being studied for $\mathrm{MG}$ in Germany. Schenider et al reported its use in 2 patients with severe refractory MG and one patient with MG-polymyositis syndrome (Schneider et al 2001). Patients received 1.5 to $2.0 \mathrm{~g}$ MMF daily. The first patient with refractory MG had previously failed combination immunotherapy consisting of cyclosporine, methotrexate, azathioprine, plasma exchange, and oral corticosteroids. This patient was placed on MMF in combination with weekly plasma exchange and corticosteroid use. Weakness reportedly improved and the patient was able to discontinue corticosteroid use and plasma exchange within 3 months of MMF initiation with no recorded relapses in the following 18 months. 
A second refractory MG patient also reported symptomatic remission while on MMF. This patient had previously failed a course of azathioprine and corticosteroids. He was subsequently given plasma exchange and started on MMF with remission achieved after 6 months.

The last patient carried the diagnosis of MG-polymyositis syndrome. After experiencing a minimal resolve of her symptoms with corticosteroids, azathioprine, cyclosporine, and plasma exchanges, the patient was given MMF in place of her azathioprine. While on these multiple agents, the patient experienced a resolve of her polymyositis symptoms within 6 months and was able to completely discontinue the use of her corticosteroids after 14 months. While on MMF, this patient also developed a hemolytic anemia which responded to a MMF dose reduction of $2.0 \mathrm{~g}$ a day to $1.5 \mathrm{~g}$ a day.

In a retrospective analysis of the use of MMF in $85 \mathrm{MG}$ patients (Meriggioli et al 2003), patients were included in the study if they: 1) Carried the diagnosis of MG; 2) Had been treated at one of two major university centers; and, 3) Had taken MMF for at least 3 months within the period February 1999 to September 2002. A determination of efficacy was based on the Myasthenia Gravis Foundation of America's (MGFA) post-intervention status scale. Of the study participants, 48 patients had previously undergone a thymectomy, 62 were on corticosteroids, 11 were on cyclosporine, 7 were on azathioprine, and 1 was on methotrexate. For all indications, $73 \%$ of patients showed improvement while $89 \%$ of patients taking MMF as the only therapy showed improvement. Eighty-one percent of patients improved with MMF as an adjunctive therapy; however, only $43 \%$ of patients with refractory MG improved with MMF. Thirty-six of the 62 patients taking simultaneous steroids were able to decrease their dose, and $57 \%$ of the patients taking pyridostigmine were able to taper this medication while on MMF. The mean time to patient reported improvement was 8.8 weeks with a maximal improvement seen at 26.7 weeks. Of the 85 patients evaluated, 2 worsened with therapy, 5 discontinued MMF secondary to side effects, and 23 patients had 1 or more side effects (the most common side effects being nausea, vomiting, diarrhea or an increased rate of infection). In addition, 1 patient developed an autoimmune granulocytopenia, 1 patient had West Nile virus, and 1 patient with cardiac valve disease developed congestive heart failure while taking MMF (Meriggioli et al 2003).

In 2003, the first double-blind, placebo controlled trial was performed to evaluate the effects of MMF ( $1 \mathrm{~g}$ twice a day) versus placebo in 14 symptomatic stable MG patients over a 5-month period (Meriggioli et al 2003). Study participants were required to have persistent daily symptoms of fatigable weakness involving the ocular, bulbar, or limb muscles. Patients who had taken azathioprine, who were pregnant, or who had purely ocular MG, severe bulbar MG or were in crisis were excluded from the study. Patients were also required to be on a stable dose of prednisone or cyclosporine and have a stable baseline QMG score prior to entry. The primary measure of efficacy was a change in QMG score while on treatment. QMG improved in the MMF group by an average of 2.86 points compared with 0.29 for placebo; however, this result was not statistically significant $(\mathrm{p}=0.30)$. A statistically significant change in SFEMG values was noted between groups $(p=0.03)$, suggesting its potential use as an early biomarker to evaluate response to treatment (Meriggioli and Rowin 2003). Changes in manual muscle testing and AChR Ab did not reach statistical significance between the two groups. Patients in the MMF group had side effects that included: diarrhea ( 2 patients), insomnia (1 patient), and urinary tract infections (2 patients) (Meriggioli et al 2003).

Two years later, a 5-patient case series of Asian patients was published on the use of MMF as an adjunctive therapy for refractory MG (Prakash et al 2007). All 5 patients had moderate to severe myasthenia symptoms, and were on corticosteroids, azathioprine, and pyridostigmine at the time of MMF initiation ( $500 \mathrm{mg} 3$ times a day). Within 2 to 4 months of MMF initiation, all patients demonstrated symptomatic improvement with maintained benefits ranging from 11 to 24 months. In addition, all patients were able to reduce their corticosteroid dosage without any clinical relapse. With the exception of one patient who experienced initial nausea and diarrhea, no patient had any significant side effects from the MMF during this study (Prakash et al 2007).

MMF has been proposed as an alternative therapy for $M G$ in patients who experience significant side effects from other therapies. In a reported case series, Lim et al (2007) demonstrated that MMF could be successfully substituted for cyclosporine in myasthenia patients with cyclosporineinduced nephrotoxicity. Two of 3 patients who switched to MMF experienced no neurological worsening of their MG with marked improvement of renal function once off cyclosporine. A third patient experienced improved renal function, but had one significant myasthenia exacerbation during MMF use (Lim et al 2007).

MM has also been studied in patients with purely ocular symptoms (ocular MG) (Chan 2008). In a prospective observational study, 31 patients with ocular MG and positive $A C h R$ 
$\mathrm{Ab}$ were identified and evaluated. Each of these patients had been started on 40 to $60 \mathrm{mg}$ of prednisone and MMF to a titrated dose of $1.0 \mathrm{~g} /$ day. Once ocular symptoms resolved, each patient's prednisone dose was tapered over a four week span. Researchers found that $87 \%(27 / 31)$ patients were able to tolerate the MMF. Of this group, 93\% (25/27) remained at (MGFA) class I for an average observation period of 4.2 years. Although there was no direct comparison group in this study, the rate of decompensation to generalized myasthenia was thought to be equal or lower than ocular myasthenia patients taking azathioprine and/or corticosteroids compared with a separate retrospective study (Sommer et al 1997; Chan 2008). Of the 4 patients who discontinued MMF during Chan's study, 3 cited persistent diarrhea while 1 discontinued the medication secondary to cost.

After much anticipation, in 2008 the results of 2 large scale, double-blind, placebo-controlled, randomized clinical trials were published which tested the efficacy of MMF in MG (Sanders et al 2008; The Muscle Study Group 2008). The relatively surprising findings from these studies have caused much discussion and deliberation in the neuromuscular community.

The first of these studies was implemented by the Muscle Study Group (MSG) and was designed to compare the use of MMF in combination with prednisone (20 mg/day) versus prednisone (20 mg/day) alone for generalized MG (The Muscle Study Group 2008). This study was an investigator-initiated, prospective, multi-centered, randomized, double-masked, and controlled to compare 2 treatment options. To be enrolled, each patient had to be over 18 years, have positive $\mathrm{AChR} \mathrm{Ab}$, and have generalized MG of mild to moderate severity. Patients were excluded if they had previously used a non-corticosteroid immunomodulating agent, had a fluctuating pyridostigmine dosage 2 weeks before enrollment, had a previously diagnosed thymoma, had severe weakness or oropharyngeal/respiratory symptoms, had a thymectomy within 12 months, or if they had used corticosteroids, PLEX, or IVIG within 90 days before enrollment.

Eighty patients were randomized to receive either $1.25 \mathrm{~g}$ of MMF twice a day and prednisone (20 mg/day) or placebo and $20 \mathrm{mg}$ /day of prednisone. Patients were followed for 12 weeks then were given the option to take open-label MMF for an additional 6 months. The primary outcome variable was the total QMG score from baseline to 12 weeks with secondary outcome variables including the change in total myasthenia MMT score, change in MG activities of daily living score, change in forced vital capacity, and change in SF-36v2 score.
Results of this study found no difference between groups in the primary and secondary outcome measures. Side effects, such as diarrhea, abdominal cramping, nausea, and vomiting, were more common in the MMF group than the placebo group. Episodes of urinary tract infection and cellulites occurred in the MMF arm of the study, however, the rate of infection did not appear to be significantly different between the two groups (The Muscle Study Group 2008).

The second study set out to assess the efficacy, safety, and tolerability of MMF as a steroid-sparing agent in patients with MG (Sanders et al 2008). Similarly to its companion study, this study was prospective, randomized, multicentered, and double-blinded. One-hundred and seventy-six patients with generalized $\mathrm{MG}$ and elevated $\mathrm{AChR} \mathrm{Ab}$ were treated with at least 4 weeks of prednisone $(20 \mathrm{mg}$ /day or greater) without additional immunosuppressive therapy before being randomized. Patients were then randomized to receive 36 weeks of prednisone and MMF at $2 \mathrm{~g}$ /day or prednisone and placebo. The primary endpoint was a composite measure defined as achievement of minimal manifestation or pharmacologic remission with reduction of corticosteroid dose on a set schedule (Sanders et al 2008). Secondary endpoints included quality-of-life scores, disease severity, and safety.

This study found that treatment with MMF was not superior to placebo during a steroid taper. There were no significant differences in patient scores for QMG, SF-36, MG activities of daily living, or global assessment between the MMF and placebo groups. In addition, serious infections occurred more frequently in the MMF group (8.0\%) compared with the placebo group (3.4\%). One of these serious infections included a fatal pneumonia in a patient taking MMF. Headache was also more frequently recorded in the MMF group (12.5\% versus 6.8\%); however reports of worsening of MG was more frequent in the placebo (20.5\%) versus the MMF (11.4\%) group (Sanders et al 2008).

\section{Discussion}

The collection of past research on MMF's role in $\mathrm{MG}$ treatment is neither exhaustive nor conclusive. Early reports and studies appear to hint at MMF's efficacy, while the latest placebo-controlled trials did not definitively prove its benefit compared with placebo. Each of the above described articles is not without criticism. Early case reports are thought provoking but are subject to positive study bias. It is not known how many times MMF was previously used for MG without effect. Such "failures" are typically not submitted to or accepted by major publications. In addition, significant 
confounding factors exist in many of the early publications. Most patients in these studies had a history of prior or coexisting treatments including corticosteroid use, azathioprine, cyclosporine, thymectomy, IVIG, pyridostigmine, or full body radiation. In many cases these therapies were being used or withdrawn during the evaluation period of MMF thus complicating the interpretation of results. In cases where a therapy had been discontinued prior to MMF use, it is possible that long term, or even delayed effects could have been inappropriately attributed to MMF. Likewise, it is possible that positive or negative interactions occurred between MMF and simultaneously used therapies. Unfortunately, none of the early studies were designed to evaluate such interference effects.

Much scrutiny has been placed on the latest randomized clinical trials due to their relatively unexpected (and perhaps contradictory) findings. Many clinicians and scientists have pondered how a medication that seemed so promising in preliminary reports could produce such a bland treatment response. Varied explanations have been proposed, such as criticisms on the: 1) Length of the studies; 2) Selected patient populations; 3) Concurrent use of prednisone; 4) Multicenter approach; and, 5) The primary efficacy measures. Many clinicians have adamantly defended MMF's use based on prior clinical experience while dismissing the results of the latest two clinical trials. The scientific and clinical communities should be careful not to completely disregard the results of these latest two trials. Instead, these trials should be accepted for what they are: two pieces of evidence in a rich tapestry of knowledge on MMF's use for MG.

In some aspects it is not overly surprising that the latest recorded effects of MMF in MG were negative. While the prior case reports and retrospective studies hinted at efficacy, the only previous placebo-controlled trial (while underpowered) did not demonstrate positive results (Meriggioli et al 2003). In addition, it is possible that the latest clinical trials did not evaluate the myasthenia patients that would have experienced the greatest benefit from taking MMF. While the Muscle Study Group's trial was well planned, carefully executed, and adequately analyzed, it only addressed the question of MMF's usefulness in a very small subpopulation of MG patients, over a very specific (and perhaps too short) time frame, in the context of both concurrent prednisone use and a specific (and perhaps understudied) primary endpoint. While some literature suggests the early efficacy of MMF, the retrospective study of 85 patients reported a maximal objective benefit only at an average of 26.7 weeks (Meriggioli et al 2003). It is possible that a separation in efficacy would have been demonstrated had the trial been carried out for a longer period of time. Also, while quantifiable, few would argue that the QMG score is the perfect measure of therapy effectiveness. The MSG study was powered to detect a difference of 3 points on the QMG score between the two arms. It is difficult to know for certain if this was the most appropriate clinical difference in which to base an outcome measure.

Likewise, there are similar limitations in the 2008 Sanders et al MMF clinical trial. As pointed out in their paper, it is possible that the results of their study were tempered by an overly rigorous definition of treatment response, a study period that was perhaps too short (36 weeks), the unexpected effectiveness of low dose prednisone $(7.5 \mathrm{mg} /$ day $)$, and an inclusion criteria that favored mild disease, older patients, men, and longstanding disease (over 3 years) (Sanders et al 2008). Like the Muscle Group's study, the results of this trial should only be extrapolated to the select MG population that shares a profile with those studied in this clinical trial.

Another possible explanation for the negative results of the 2008 clinical trials is that the benefit of low dose prednisone and MMF on moderately affected MG patients is not additive. MMF was not studied in isolation, but rather as an add-on therapy to prednisone. Further studies on the efficacy of MMF as a monotherapy (against placebo) or as an add-on therapy to other non-steroid immunosuppressive therapies should be performed to address this hypothesis.

In the future, the use of MMF should be studied in a more generalizable myasthenia population. It is possible that the study populations in the negative clinical trials had such mild cases that an add-on medication would not produce any additional measurable benefit. For this reason, MMF should also be studied in patients with more severe MG or with $\mathrm{MG}$ refractory to corticosteroid treatment alone.

Lastly, it should be noted that a large discrepancy exists in the end point selection in most of the above listed studies. It is likely that this variation in outcome measure selection has played a significant role in the apparently conflicting results of the above literature. Continued work will be required to fully identify and validate the ideal clinical outcomes for measuring changes in patient-relevant MG symptoms during future clinical studies.

Based on the most recent studies, difficult questions will soon be asked by the patients, insurance companies, and the public. The use of MMF for MG will likely be questioned. Physicians will have to decide on the best course of action for each individual patient based on what is currently available in the literature. Additional studies are required given the 
limitations of the last two negative clinical trials and the many unanswered questions regarding MMF's use for MG.

\section{Conclusions}

Much work remains to determine MMF's true place in MG management. Despite recent studies, questions still exist regarding MMF's long-term efficacy, optimal dose, optimal population of use, optimal length of use, benefit as a combination therapy, and long-term safety profile. These specific questions should be addressed via future prospective placebo-controlled studies.

\section{Disclosures}

The authors have no conflicts of interest to disclose.

\section{References}

Allison A. 2005. Mechanisms of action of mycophenolate mofetil. Lupus, 14(Suppl 1):s2-8.

Allison A, Eugui EM. 2005. Mechanisms of action of mycophenolate mofetil in preventing acute and chronic allograft rejection. Transplantation, 80(2 Suppl):S181-90.

Amato AA, Griggs, R.C. 2003, Treatment of idiopathic inflammatory myopathies, Current opinion in neurology, 16(5): pp 569-75.

Cahoon WD Jr, Kockler DR. 2006. Mycophenolate mofetil treatment of myasthenia gravis. Ann Pharmacother, 40:295-8.

Chan JW. 2008. Mycophenolate mofetil for ocular myasthenia. J Neurol, 255:510-3.

Chaudhry V, Cornblath DR, Griffin JW, et al. 2001. Mycophenolate mofetil: a safe and promising immunosuppressant in neuromuscular diseases. Neurology, 56:94-6.

Ciafaloni E, Massey JM, Tucker-Lipscomb B, et al. 2001. Mycophenolate mofetil for myasthenia gravis: an open-label pilot study. Neurology, 56:97-9.

Ciafaloni E. 2005. Mycophenolate mofetil and myasthenia gravis. Lupus, 14(Suppl 1):s46-9.

Draper HM. 2008. Depressive disorder associated with mycophenolate mofetil. Pharmacotherapy, 28:136-9.

Enk AH, Knop J. 1997. Treatment of pemphigus vulgaris with mycophenolate mofetil. Lancet, 350:494.

Epinette WW, Parker CM, Jones EL. et al. 1987. Mycophenolic acid for psoriasis. A review of pharmacology, long-term efficacy, and safety. J Am Acad Dermatol, 17:962-71.

European Mycophenolate Mofetil Cooperative Study Group. 1995. Placebo-controlled study of mycophenolate mofetil combined with cyclosporin and corticosteroids for prevention of acute rejection. Lancet, 345:1321-5.
Hauser RA, Malek AR, Rosen R. 1998, Successful treatment of a patient with severe refractory myasthenia gravis using mycophenolate mofetil. Neurology, 51:912-3.

Larkin G, Lightman S. 1999. Mycophenolate mofetil. A useful immunosuppressive in inflammatory eye disease. Ophthalmology, 106:370-4.

Levin N, Mali A, Karussis D. 2005. Severe skin reaction related to mycophenolate mofetil for myasthenia gravis. Clin Neuropharmacol, 28:152-3.

Lim AK, Donnan G, Chambers B, Ierino FL. 2007. Mycophenolate mofetil substitution for cyclosporine-dependent myasthenia gravis and nephrotoxicity. Int Med J, 37:55-9.

Meriggioli MN, Ciafaloni E, Al-Hayk KA, et al. 2003. Mycophenolate mofetil for myasthenia gravis: an analysis of efficacy, safety, and tolerability. Neurology, 61:1438-40.

Meriggioli MN, Rowin J, Richman JG, et al. 2003. Mycophenolate mofetil for myasthenia gravis: a double-blind, placebo-controlled pilot study. Ann N Y Acad Sci, 998:494-9.

Meriggioli MN, Rowin J. 2000. Treatment of myasthenia gravis with mycophenolate mofetil: a case report. Muscle Nerve, 23:1287-9.

Meriggioli MN, Rowin J. 2003. Single fiber EMG as an outcome measure in myasthenia gravis: results from a double-blind, placebo-controlled trial. J Clin Neurophysiol, 20:382-5.

Neurath MF, Wanitschke R, Peters M, et al. 1999. Randomised trial of mycophenolate mofetil versus azathioprine for treatment of chronic active Crohn's disease. Gut, 44:625-8.

Nowack R, Gobel U, Klooker P, et al. 1999. Mycophenolate mofetil for maintenance therapy of Wegener's granulomatosis and microscopic polyangiitis: a pilot study in 11 patients with renal involvement. $J \mathrm{Am}$ Soc Nephrol, 10:1965-71.

Prakash KM, Ratnagopal P, Puvanendran K, et al. 2007. Mycophenolate mofetil - as an adjunctive immunosuppressive therapy in refractory myasthenia gravis: The Singapore experience. J Clin Neurosci, 14:278-81.

Rozen TD. 2004, Complete but transient relief of chronic cluster headache with mycophenolate mofetil. Headache, 44:818-20.

Sanders DB, Hart IK, Mantegazza R, et al. 2008. An international, phase III, randomized trial of mycophenolate mofetil in myasthenia gravis, Neurology, 71:401-6.

Schneider C, Gold R, Reiners K, et al. 2001, Mycophenolate mofetil in the therapy of severe myasthenia gravis. Eur Neurol, 46:79-82.

Schneider-Gold C, Hartung HP, Gold R. 2006. Mycophenolate mofetil and tacrolimus: new therapeutic options in neuroimmunological diseases. Muscle Nerve, 34:284-91.

Sommer, N, Sigg, B, Melms, A, et al. 1997. Ocular myasthenia gravis: response to long-term immunosuppressive treatment. J Neurol Neurosurg Psychiatry, 62:156-62.

The Muscle Study Group. 2008. A trial of mycophenolate mofetil with prednisone as initial immunotherapy in myasthenia gravis. Neurology, 71:394-9.

Vernino S, Salomao DR, Habermann TM, et al. 2005. Primary CNS lymphoma complicating treatment of myasthenia gravis with mycophenolate mofetil. Neurology, 65:639-41. 
